\title{
A GRAPH-MATCHING APPROACH TO INDOOR LOCALIZATION USING A MOBILE DEVICE AND A REFERENCE BIM
}

\author{
F. J. Bot ${ }^{1}$, P. Nourian ${ }^{2}$, E. Verbree ${ }^{3}$ \\ ${ }^{1}$ Sweco Netherlands, GIS\&ICT, Geomatics, Fanny.Bot@sweco.nl \\ 2 Delft University of Technology, Faculty of Architecture and the Built Environment, Design Informatics, P.Nourian@tudelft.nl \\ ${ }^{3}$ Delft University of Technology, Faculty of Architecture and the Built Environment, GIS Technology, E.Verbree@tudelft.nl
}

KEY WORDS: Indoor Localization, Spectral Graph Analysis, Graph-Matching, VI-SLAM

\begin{abstract}
:
The presented method provides for a possibility to perform graph-based indoor localization, by comparing the topological structure embedded in a mesh model to the topological structure of a semantically rich reference model, specifically a BIM. However different in nature and structure, both input sources can be converted to a graph of similar calibre, such that they can be tested for a match. After a match between both graphs is found, the current position of the actor within the mesh model can be translated to the room found in the graph. This room is now connected to a room within the reference graph, for which the semantics are stored in the BIM. Returning these to the actor, a location description can be formed.
\end{abstract}

Many indoor positioning methods have been developed, which can provide an actor with a relative geometric place. Most preferred are positioning systems not relying on a contingent system, which can be performed using a fusion of sensors embedded into a mobile device. Such a system found to perform sufficiently is VI-SLAM, simultaneously building a geometric place and tracking each pose and heading relatively. Its output is a mesh model, in which a viewshed of the indoor environment is built. Indoor localization however, entails finding a humanly interpretable qualitative description of a place, rather than a quantitative position in Euclidean space. In order to transcend such a position to a meaningful location, the context of an indoor environment has to be understood. As the interpretation of indoor space is different across the board, misalignment in data sources representing the indoor environment occurs.

\section{INTRODUCTION}

Indoor localization is a widely researched subject (Xiao et al., 2016, Yassin et al., 2016, Zafari et al., 2017, Zlatanova et al., 2013), as the complexity and size of many public buildings require extensive and properly designed methods to facilitate location specific processes (Mautz, 2012, Lemmens, 2013, Zlatanova et al., 2014). The methods created for such services outdoor cannot be translated directly, new definitions of each component need to be defined (Mautz, 2012). A proper localization process requires positioning into a semantically rich model of the physical environment, or the simultaneous generation of both (Fuentes-Pacheco et al., 2012, Lemmens, 2013). In other words, the context of an indoor environment has to be understood, such that a position can be transcended to a meaningful location.

The target user for such a process would be any actor executing any type of task inside a public building, thus an indoor localization system should be made easily attainable to anyone. As the use of smartphones is widespread, the capabilities of such devices lend themselves for outdoor localization as well, and many Location Based Systems (LBS) are specifically aimed at smartphones, ideally such a system would be designed in order to function on a mobile device.

Thus, a mobile indoor positioning system should be applied, along with the availability of a contextual 3D building model to position the actor in, so that the combined information defines the actors location. An autonomously operating process deemed sufficient for indoor positioning is Simultaneous Localization and Mapping (SLAM), especially when based on the integration of several different sensors (Fuentes-Pacheco et al., 2012, Zlatanova et al., 2013, Zafari et al., 2017). If an actor's position can be integrated into a contextual map of indoor space, the location can be determined. Such a map should be comprised of geometry, topology and semantics (Isikdag et al., 2013). The geometry then represents the physical boundaries of indoor space, while the topology describes the connectedness and nearness of the elements that form these boundaries. Semantics contain a functional description of indoor space and its boundaries, which functions as a context that can transcend a position into a location.

An autonomous SLAM process can output a polygonal mesh created on-the-fly as a model of indoor space, containing its geometry and topology. The output model is used as a map in which an actor can be positioned. Semantics describing building geometry and topology are generally stored in a Building Information Model (BIM). The placement of a real-time position of the actor operating the hand-held device into the semantically rich map would generate an indoor location.

The aim of the presented method is to develop a method to teach a mobile device to understand its surroundings. This is to be reached by capturing the context and all of its relevant meaning, in order to transcend data to information. As the relevance of information is application dependent (Afyouni et al., 2012), it should be grouped and retrievable by a single marker, i.e. to find a location would be to find the an ID connected to information about the room an actor is in. Therefore, an established standard should be used to store contextual information, so that each application can be type-focused and be built upon the same 
principles. Then, the accessibility of contextual information while on location enables a wide range of possible applications. The main objective on a data level would then be to generate this single marker as a representation of the connection between an indoor environment and an information source describing its context. A method to perform this process is proposed, which is built on best practices and underlying principles, taken from an interdisciplinary viewpoint. See Figure 1 for an overview of the explored research fields.

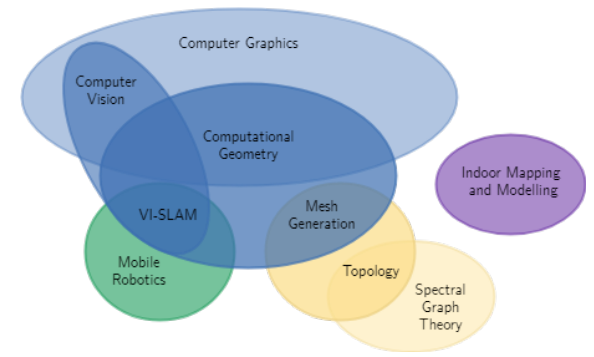

Figure 1. Euler diagram of fields that apply to the theoretical framework underlying the presented method.

\section{TERMINOLOGY}

In order to integrate theory from varying research fields, an alignment in terminology is necessary. Thus a concise definition of indoor space is needed, in order to transcend a geometrically defined position to a human interpretable location. Intuitively, we may perceive space as what cannot be seen or touched, as what is left when every other instance around is defined. The term space has become an abstraction of everything outside of the boundaries of everything else. In mathematics and philosophy, space has become what defines the boundaries of everything embedded within. A definition more fitting to this description would be an "expanse in which everything is located" (Wolfram-Alpha, 2019), or even "that, which results from places taken together" (Leibniz et al., 2000). Thus, a space represents a structure, often a set with relational properties, to which all its members adhere. The characteristics of a type of space provide the context in which objects can be examined and represented (Huisman, de By, 2009, Marel, 2016), i.e. providing a type of grid on which embedded elements can be represented. Two specifically interesting classes of mathematical space are topological and metric space.

\subsection{Topological Space}

Topology describes the relationships between (parts of) objects, that do not change under continuous deformation (Huisman , de By, 2009). As these relations are intrinsic, and thus invariant of ambient space, topology studies the construction of an object, rather than its shape. It can be used to "abstract the inherent connectivity of objects while ignoring their detailed form" (Weisstein, 2019c). A Topological Space is devoid of geometric structure, so that the relations and characteristics of objects can be examined qualitatively. The topology $T$ on set $X$ then defines topological space $(X, T)$. A Manifold can be seen as a topological space, which locally resembles Euclidean space (Edelsbrunner, Harer, 2010, Rowland, 2019). The topology of a set can be simplified to a graph structure $\mathcal{G}$, embedded in a Network Space that defines the topological connectedness between its members using an unordered set of node pairs or links. A $\operatorname{Graph} \mathcal{G}=(\mathcal{N}, \mathcal{L})$ is a set of nodes pairwise connected by links which abstractly represent the connectivity between elements (Nourian, 2018, Cromley, 1989, Worboys , Duckham, 2004). The dual $\mathcal{G}^{*}$ of a graph can be constructed by translating $k$-dimensional features to $n-k$-dimensional features in $\mathbb{R}^{n}$ (Nourian, 2016, Weisstein, 2019a).

\subsection{Metric Space}

Metric Space is "a set with a global distance function, that for every two of the set's points gives the distance between them as non-negative real number" (Weisstein, 2019b), or the structure in which distances between all members can be defined (Worboys, Duckham, 2004). A metric, as ordered pair $(S, d)$ can be imposed on a set $S$ of arbitrary type, by defining how a shortest distance $d$ between two members can be calculated. A structure is a complete metric when this shortest distance between two points is defined by a straight line, i.e. $d(\mathbf{s}, \mathbf{t})=|\mathbf{t}-\mathbf{s}|, \mathbf{s}, \mathbf{t} \in \mathbb{R}^{n}$. Metric space has a natural topology based on the notion of proximity, as for each point a set of nearest neighbours can be found based on distance, and such a neighbourhood forms an open set (Munch, 2017, Worboys , Duckham, 2004). The complete metric set of which all members are directly related in terms of distance and angle, is called the Euclidean Space $\mathbb{E}^{n}$ (Worboys , Duckham, 2004). It contains all $n$-tuples of real numbers (Stover , Weisstein, 2019) and provides for an intuitive abstraction of physical space without a specific origin. It defines the structure of the real vector space $\mathbb{R}^{n}$ of the same dimension.

\subsection{Indoor Space}

Often, a space is defined in terms of either place, boundaries, or function. A place may be a geographic space that has a potion on the face of the earth (Huisman, de By, 2009). Furthermore, a place "is that, which is the same in different moments to different existent things" (Leibniz et al., 2000). In the OGC CityGML standard, indoor space is inside one or multiple buildings, bounded by architectural components (Lee et al., 2016). The IFC BIM standard (ISO 16739) mentions enclosure of an area, as well as the fact that a specific human activity can be executed in a space. This space is bounded either by a functional transition, or a geometrical border. However, mathematically speaking, a space dictates the type of properties elements embedded within may have. A single type of space representation does not exclude the possibility of mapping an object into another. The physical world may then be represented in several dimensions of ambient space, as to capture its complexity (Ekholm, Fridqvist, 2000, Zlatanova et al., 2014).

Pure positioning entails finding a point or area occupied by a physical object or person (Mautz, 2012, Groves, 2013, Sithole , Zlatanova, 2016). This term is most used as to find a position $\mathbf{p}=(X, Y, Z)^{T} \in \mathbb{R}^{3}$, relative to the systems' origin. Contrasting to the quantitative position, a location entails finding a qualitative description of the occupied area. Providing semantic context for a position would then allow an actor to find a location with a specific meaning, or to perform localization. It requires topological correctness of sensors used to determine the underlying position, and precise accuracy of is generally of less importance (Mautz, 2012). Based on either position or location, an actor can execute navigation, as determining heading and velocity of the current trajectory, and being guided along an optimal path to the destination (Mautz, 2012, Groves, 2013, Sithole , Zlatanova, 2016). Figure 2 exemplifies these principles. 

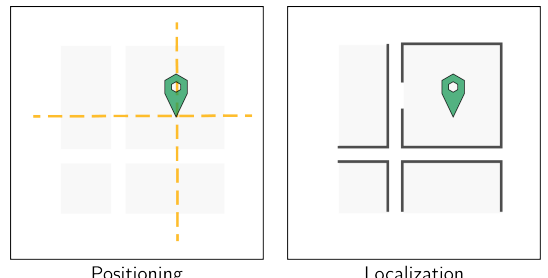

Localization

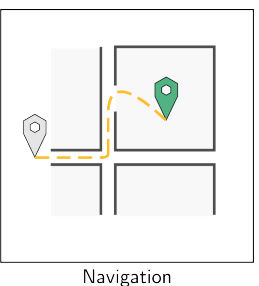

Navigation
Figure 2. Differences between positioning, localization and navigation for indoor situations.

A full representation of indoor space would allow an actor to execute activities as positioning, localization and navigation, which would require geometric, topological and semantic information (Isikdag et al., 2013). The complex indoor space can thus be modelled threefold: the geometric place can be modelled in Euclidean space, with positions $(X, Y, Z)^{T} \in \mathbb{R}^{3}$, as the formal representation of an object (Worboys, Duckham, 2004). The connectivity and boundaries can be modelled in topological space as a primal 2-manifold surface $\mathcal{S}$ and its dual graph $\mathcal{G}^{*}$. The function of each object can be modelled in semantic space, as a set of characteristics defining context and facilitation of human activity. As such, indoor space can be occupied by elements, compliant to the rules of the structure.

\section{METHODOLOGY}

Performing indoor localization on a mobile device is interpreted as a data matching problem, where a mesh of an indoor environment is compared to a reference model. This is done by extracting a graph from both input sources, and comparing these to form a match (Figure 3). First, the input sources are analysed. Then follows the extraction of topological graphs from both geometric models. As both input models are based on different data types derived from different interpretations of indoor space, both require a different method for extracting the graph. In both cases, the resulting graph represents a network of rooms, of which the intrinsic properties are analysed by computing the spectral map. Thus the analysed geographic indoor location, represented in a geometric model, is further abstracted to a topological, then graph and then spectral model (Nourian et al., 2016).

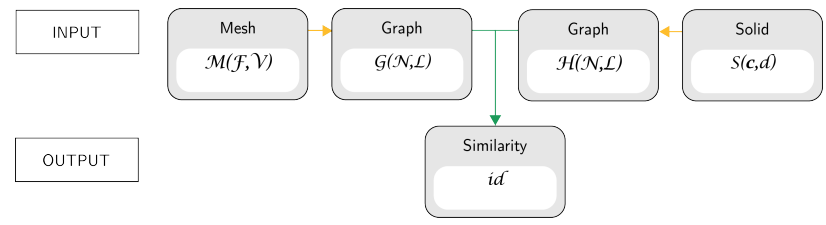

Figure 3. Methodological workflow for creating a match between a VI-SLAM mesh and a reference BIM library of solids. Graphs are extracted from both input types (yellow arrows), which are compared and matched based on similarity (green arrow).

The input mesh is captured using Visual-Inertial SLAM (VI-SLAM), an autonomously operating positioning system, reliable due to the integration of various sensors (Fuentes-Pacheco et al., 2012, Groves, 2013). The use of cameras integrated into a mobile device furthermore makes VI-SLAM attainable, affordable, portable, low on power consumption, and intuitively appealing (Riisgaard, Blas, 2003). Developed in the field of mobile robotics, the SLAM process tracks a position $\mathbf{x}_{k} \in \mathbb{R}^{3}$ relative to former positions, the heading $\mathbf{v}_{k}$ chosen from each position, and the relative position $\mathbf{m}_{i}$ of each landmark in sight (Bailey, Durrant-Whyte, 2006). These landmarks, as sharp edges and corners in the environment, are captured frame by frame, and stitched together into a viewshed along the traversed trajectory. Thus, rather than localization, SLAM executes simultaneous positioning and mapping. The input BIM model is built according to IFC standards, and assumed to contain standard case geometry, and sufficient functional attribution. For the purposes of testing a matching method, both models contain the same amount of rooms, though in real adaptation the BIM would contain a full building (level) of which a part would be matched with the graph extracted from the mesh.

\subsection{Graph Extraction from Mesh}

The remapping of mesh $\mathcal{M}(\mathcal{F}, \mathcal{V})$ into graph $\mathcal{G}(\mathcal{N}, \mathcal{L})$ requires first extracting the dual graph, after which it is simplified to a graph representing rooms.

The dual graph is constructed based on face to face adjacency, as extracted from the .obj file structure the input mesh is stored in (Figure 4). Then, each primal face normal is computed assigned to its dual node. This attribute is used in a connected components algorithm, where two nodes are accepted into the same region if the dot product similarity of both face normals does not fall below a set threshold. The connected components algorithm performs a depth first search through all nodes stored in $\mathcal{G}$, and returns regions representing similarly oriented partitions of the input mesh. Region size is used to filter out clutter. Furthermore, only the largest horizontally oriented surface is kept, as floor partition. This is tested using the dot product similarity to unit vector $\hat{\mathbf{k}}=(0,0,1)^{T}$. The result is a classification of $\mathcal{G}$ into a floor region and several wall regions.
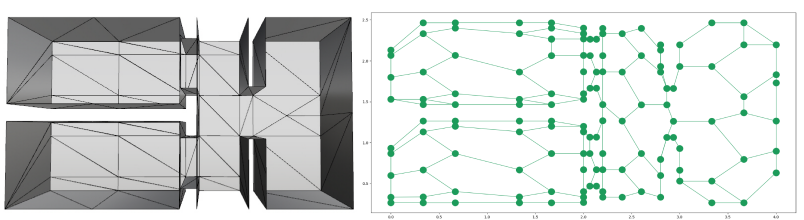

Figure 4. Mesh $\mathcal{M}$ of the test dataset and its dual graph $\mathcal{G}$.

In order to analyse the intrinsic properties of $\mathcal{G}$, its spectral map is computed. This is based on the graph Laplacian, constructed as:

$$
\mathbf{L}_{i, j}=\left\{\begin{aligned}
d_{i} & \text { if } \quad i=j \\
-1 & \text { if } \quad(i, j) \in \mathcal{L} \\
0 & \text { otherwise }
\end{aligned}\right.
$$

where $\quad \mathbf{L}_{i, j}=$ Laplacian operator over node pairs $(i, j) \in \mathcal{G}$ $d_{i}=$ degree of node $i$

$$
\mathcal{L}=\text { set of links }(i, j) \in \mathcal{G}
$$

Its spectrum of eigenvalues $0=\lambda_{1} \leq \lambda_{2} \leq \cdots \leq \lambda_{n}$ allow for a simplification as $\mathbf{L x}=\lambda \mathbf{x}$, corresponding with eigenvectors $\mathbf{x}_{1}, \ldots, \mathbf{x}_{n}$. The number of eigenvalues which return $\lambda_{i}=$ 0 corresponds with the number of connected components in the graph. Furthermore, the second smallest eigenvalue $\left(\lambda_{2}\right)$ provides for a particularly interesting interpretation of a graph. The graph is only connected if $\lambda_{2} \geq 0$, and the further from zero, the stronger the graph is connected. Its corresponding eigenvector $\mathbf{x}_{2}$, the Fiedler vector, gives information about 
connectivity (Chung, 1996, Spielman, 2007, Luxburg, 2007). The sign of its values may be interpreted as a division of the data into parts according to its sparsest cut, or the cut for which the least edges are broken. Such a cut can be stated as:

$$
\operatorname{cut}(A, B)=\sum_{i \in A, j \in B} s_{i j}
$$

where

$$
s_{i, j}=\text { similarity between pair of nodes }(i, j) \in \mathcal{G}
$$

$(A, B)=$ disjoint sets of nodes $\mathcal{N}$ resulting from the cut

A threshold $t$ can always be found such that the set of edges in a cluster $S=\left\{i: \mathbf{x}_{2} \leq t\right\}$ is most optimally connected. A common solution is to find the median cut as $n_{\text {left }}<0<$ $n_{\text {right }}$, meaning to find the largest gap between values of $\mathbf{x}_{2}$ within the graph (Spielman, 2007). The result for such a cut in the test data set is visualized in Figure 5. A clustering algorithm can be constructed which optimizes the cuts to be made, in order to divide a graph into clusters (Luxburg, 2007).
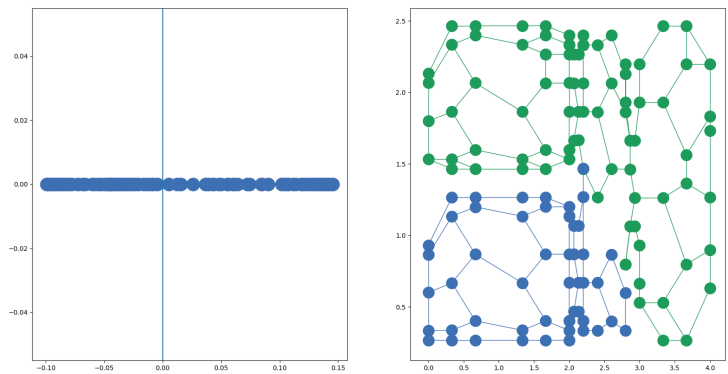

Figure 5. Median cut in $\mathcal{G}$ based on its eigenspectrum.

Alternatively to using Fiedler cuts to divide a graph, the spectral properties can be used as a basis for clustering. When mapping all nodes into a spectral embedding, the new coordinates imply clusters based not only on nearness of the faces all nodes represent, but also the connectivity between them. A spectral embedding of a graph can be given by assigning eigenvectors as coordinate scalers to node $i$, e.g. placing it at point $\left(\mathbf{x}_{2}(i), \mathbf{x}_{3}(i)\right)$. The eigenvectors are used to draw an almost always planar embedding, uniquely representing the underlying structure of the graph (Nourian, 2016, Spielman, 2007, Luxburg, 2007). A spectral and dual embedding of $\mathcal{G}$ can be found in Figure 6. Both visual representations prove an implicit occurrence of three or four clusters within the test data, of which the two rooms on the left are most strongly disconnected from the rest of the model.

The coordinates $\mathbf{x}$ of the spectral embedding of $\mathcal{G}$ can be used as input for a spectral clustering algorithm, where the coordinates are treated as nodes, for which a nearest neighbour graph is constructed (Pedregosa et al., 2011). Based on normalized graph cuts, an input is created for a k-means algorithm which assigns each data point with a cluster index. Using the eigenspectrum of this nearest neighbour graph, the number of $\mathrm{k}$ clusters is automatically determined. A large advantage of this algorithm is that it is suitable for non-convex clusters. Figure 7 shows the output of this algorithm, where each determined cluster grouped by colour, and remapped into the dual embedding of $\mathcal{G}$.
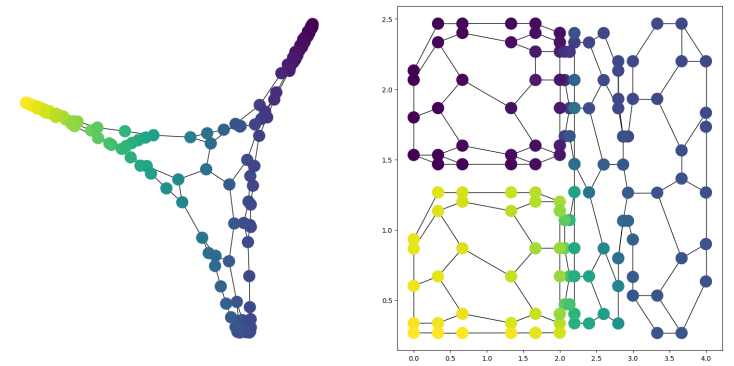

Figure 6. Spectral embedding (left) and dual embedding (right) of $\mathcal{G}$, coloured according to Fiedler vector $\mathbf{x}_{2}$.
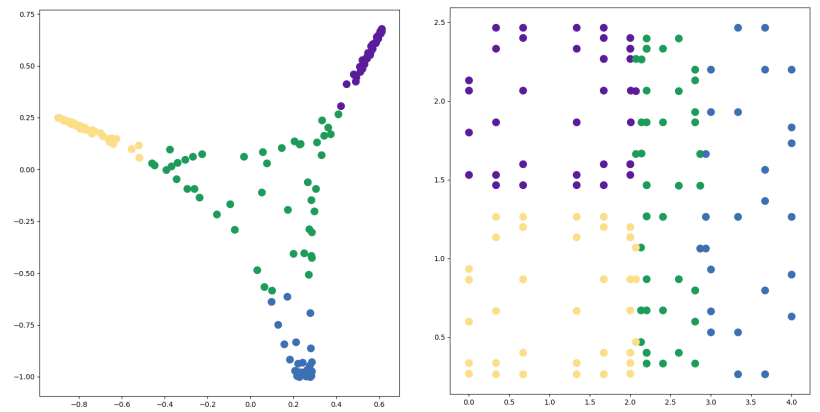

Figure 7. Result of spectral graph clustering of spectral coordinates of $\mathcal{G}$ as clusters in a spectral embedding (left) en

the output remapped to their original positions (right).

After clustering, nodes are filtered according to the extracted components and clusters. The clusters divide the single floor component into floor pieces, each now represented by a single node. These nodes are linked if the separated components are connected. For each floor component, the wall components in the same cluster are linked to it. The result as simplified graph $\mathcal{G}^{\prime}$ is visible in Figure 8. Figure 9 shows conceptual representation of the extracted graph.
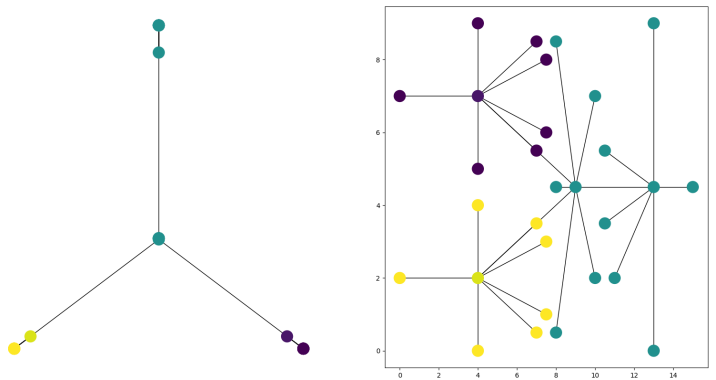

Figure 8. $\mathcal{G} /$ in its spectral (left) and positional embedding (right).

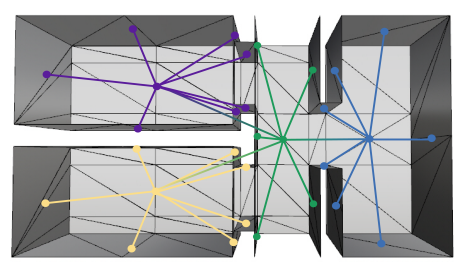

Figure 9. Conceptual representation of $\mathcal{G}$ ।. 


\subsection{Graph Extraction from BIM}

The remapping of the reference BIM into graph $\mathcal{H}(\mathcal{N}, \mathcal{L})$ requires first extracting room representations, after which a graph can be formed.

A room in a BIM model is inherently stored as an ifcSpace. A BIM space is defined as "an area or volume bounded actually or theoretically. Spaces [..] provide for certain functions within a building." (BuildingSMART, 2016). The composition of an ifcSpace is defined by its storey and placement, and defines elements within it. As such, it can be used to determine the distinction as well as the connection between rooms. A navigable graph model can be extracted by handling each ifcSpace as node (Diakité, Zlatanova, 2016). A wall, as a basic building element which can bound a partition of indoor space, is stored as ifcWall. In its standard case it is stored as a (poly)curve $c$ built by Points $\mathbf{p}_{i}=(x, y)^{T} \in \mathbb{R}^{2}$ describing the general footprint of the element, and an extrusion depth $d$. Thus, an ifcWall is stored as a solid $\mathcal{S}(c, d)$. The coordinates are defined relative to object an origin $\mathbf{O}=(X, Y, Z)^{T} \in \mathbb{R}^{3}$ and set on a reference plane. The coordinates of the origin are relative to the full model. Each ifcSpace entity may be connected with its bounding walls by relative placement. Thus, for each ifcSpace a set of ifcWall objects may be extracted. If the same wall is related to more than one ifcSpace, it should be duplicated. Furthermore, the amount of if cDoor elements embedded into the walls around an ifcSpace represent the number of links that should be laid from a single ifcSpace to one or more other ifcSpace elements. Walls bounding the same room are related by the Path Connectivity element, which is used to ensure a single material or profile definition. Thus it can be used to group walls per room. It can be applied to check if rooms assigned to spaces in the previous step are correct and complete. If after these steps any of the walls is unassigned, the coordinates embedded in the if cWall elements may be used to find their placement. Graph $\mathcal{H}$ (Figure 10) is now constructed by translating each ifcSpace to a node, and connecting it with a new node for each adjoining ifcWall. A conceptual representation of $\mathcal{H}$ can be found in Figure 11.
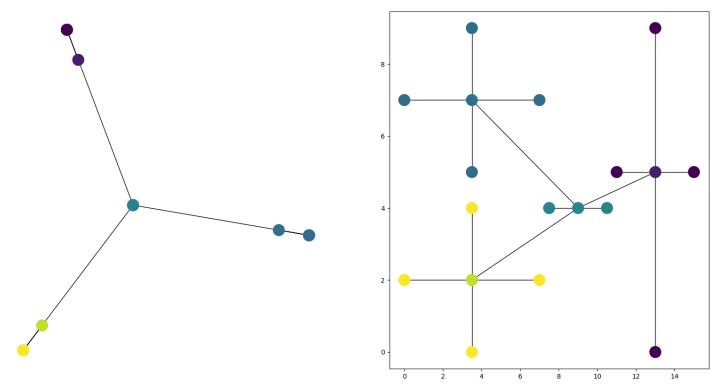

Figure 10. $H$ in its spectral (left) and positional embedding (right).

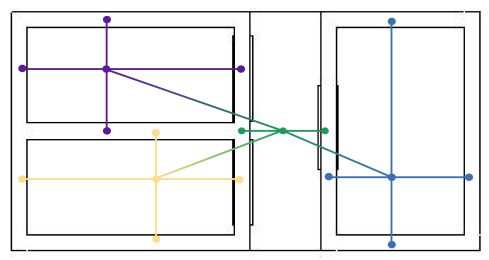

Figure 11. Conceptual representation of $\mathcal{H}$.

\subsection{Spectral Graph Matching}

Aside from embedding and clustering, spectral properties are further used to find a match between $\mathcal{G} /$ and $\mathcal{H}$. Graph matching entails finding correspondence between two given graphs, where the underlying structures are at least highly similar (Conte et al., 2004). Theoretically, $\mathcal{G} /$ and $\mathcal{H}$ should be isomorphic, as the represent the same indoor environment. However, due to differences in interpretation of indoor space, the resulting models are slightly different (compare Figures 8 and 10). The option remaining would then be to construct an inexact matching process. To further explore the application of spectral graph properties, its embedding is used as input for a method which can match co-spectral graphs, even if the number of nodes in both graphs is different. Here, clusters of nodes are compared within an embedding of all nodes into a vector subspace (Kosinov, Caelli, 2002). This re-embedding is based on the eigenspectrum of the data covariance matrix $\boldsymbol{\Sigma}$ as $\boldsymbol{\Sigma}=\mathbf{U} \boldsymbol{\Lambda} \mathbf{U}^{T}$. By using the matrix of eigenvectors $\mathbf{U}$, the projection of data point $\mathrm{x}$ can be constructed using:

$$
\hat{\mathbf{x}}=\mathbf{U}_{k}^{T} \mathbf{x}
$$

where $\quad \hat{\mathbf{x}}=$ coordinates in vector sub space

$$
\begin{aligned}
& \mathbf{U}_{k}^{T}=\text { transposed matrix of first } k \text { eigenvectors } \\
& \mathbf{x}=\text { coordinates of spectral embedding }
\end{aligned}
$$

To be come a useful operator, $\mathbf{U}$ it has to be decreased to the use of the first $k$ eigenvectors. Of these first $k$ eigenvectors, only the first few dimensions are meaningful, corresponding to the dimensionality of $\mathbf{x}$. In the described case, each data point $\mathbf{x}$ represents a $2 \mathrm{D}$ coordinate of a node in the spectral embedding of the graph (Figure 6 left). The new embedding $\hat{\mathbf{x}}$ can be used as a basis for another clustering algorithm (Figure 12). If the nodes in the same cluster are similar enough to pass as a match, it is accepted. This can be tested based on certain attributes of the nodes, e.g. the coordinates in the original graph or the spectral values.

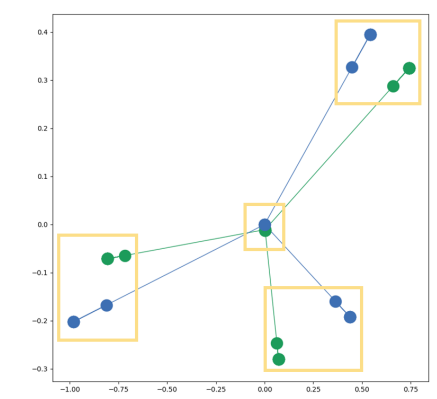

Figure 12. Clusters found in the vector subspace embedding for $\mathcal{G}$ (green) and $\mathcal{H}$ (blue). 


\section{LOCALIZATION PROCESS}

After a match between mesh graph $\mathcal{G}$ and reference graph $\mathcal{H}$ is found, its characteristics can be used as a basis for localization. In the mesh model, the actor capturing the indoor environment has a current position. As it is situated in the local coordinate system the mesh is stored in, this position can be connected to the closest room found in $\mathcal{G}$. The nodes of this room in $\mathcal{G}$ are connected to the same room in $\mathcal{H}$ through the matching process. These are attributed with a marker, e.g. an id, which refers to a set of semantics describing the characteristics of this room. Returning these to the actor, a location description is formed.

\subsection{Discussion}

A factor strongly influencing the possibility of using purely structural graphs as a basis for indoor localization, is the interpretation of indoor space lying at the basis of the model. $\mathcal{G}$ was formed based on a VI-SLAM process, in which a sufficient description of an environment is a geometrically and topologically structured viewshed of an already traversed trajectory. A set of connected points describes a surface bounding navigable space. As such, $\mathcal{G} /$ (Figure 9) shows nodes for each surface partition encountered while traversing through the environment. $\mathcal{H}$ was formed based on a BIM, in which a sufficient description of an environment entails the geometric definition of bounding solids, preferably enriched with context defining semantics. Without additional interpretation, $\mathcal{H}$ would contain a minimal amount of nodes. However, nodes representing walls are repeated when connected to several ifcSpace elements, to mimic the results of $\mathcal{G}$ (Figure 11). A difference still occurs around doors: each surface partition next to a door is turned into a node, where there would be a single if cWall in the BIM. However different in nature and structure, both input sources can be converted to a graph of similar calibre, such that they can be tested for a match. However, before such a match can be made, a number of rooms must have been visited, in order to retrieve a meaning full graph. Thus, initializing the proposed process would entail extensive traversal through an unknown building, which would not be an ideal situation for indoor localization. Alternatively, the process could be initialized by providing an actor with a marker representing the hallway or room to start from, as well as its structure relative to the two closest partitions of indoor space to be defined. Nonetheless, the topological structure underlying a mesh model captured using a mobile device is strongly similar to the topological structure that can be extracted from a BIM model representing the same indoor environment. However, neither a boundary mesh nor a volumetric BIM can fully capture the knowledge of indoor space and its topological relations (Khoshelham , Díaz-Vilariño, 2014). A culmination of different dimensionalities is necessary in order to fully represent the meaning of an indoor space (Sithole, Zlatanova, 2016, Zlatanova et al., 2014). One of the goals for the proposed method was to let go of the purely geometrical approach often applied in indoor localization methods, and utilizing another type of descriptor of the indoor space. This structural approach proves the amount of intrinsic characteristics of indoor space to be found in a good representation, while exemplifying its sole adaptation would not prove sufficient.

\section{CONCLUSIONS}

The proposed method provides for a possibility to perform graph-based indoor localization, using a mesh model created using SLAM on a mobile device and a reference BIM. The inherent organization of such a model has a great influence on how a representation of indoor space can be built, and on how it relates to the physical world. Understanding these implications by mathematically defining the type of models at hand, can aid in understanding how encountered problems can be solved. Thus, an indoor space is redefined here as a structure defining the representation of its embedded objects, such that mathematical processes can be applied to form a humanly understandable description of environments embedded into indoor space. Its aspects are defined as follows:

- Geometry - The place an actor is in

- Topology - The structure of the place the actor is in

- Semantics - The meaning of the place the actor is in.

Indoor localization then entails positioning an actor into a meaningful context. As such, the spectral analysis of the topology of indoor space allows for correct semantic assignment to indoor positions, so that an understanding of indoor space can be reached.

\section{ACKNOWLEDGEMENTS}

Thanks to Abdoulaye Diakité for his guidance during the initiation and exploration phase of this research.

\section{REFERENCES}

Afyouni, I., Ray, C., Claramunt, C., 2012. Spatial models for context-aware indoor navigation systems: A survey. Journal of Spatial Information Science, 4, 85-123.

Bailey, T., Durrant-Whyte, H., 2006. Simultaneous localization and mapping (SLAM): Part I. IEEE Robotics and Automation Magazine, 13, 108-117.

BuildingSMART, 2016. IFC4 Add2 - ifcSpace. http://www.buildingsmart-tech.org/ifc/IFC4/Add2/html/.

Chung, F., 1996. Spectral Graph Theory. CBMS Regional Conference Series in Mathematics, 92, American Mathematical Society, Providence, Rhode Island.

Conte, D., Foggia, P., Sansone, C., Vento, M., 2004. Thirty Years of Graph Matching in Pattern Recognition. International Journal of Pattern Recognition and Artificial Intelligence, 18, 265-298.

Cromley, R. G., 1989. Digital Cartography. Prentice-Hall.

Diakité, A. A., Zlatanova, S., 2016. Extraction of the 3D Free Space From Building Models for Indoor Navigation. ISPRS Annals of Photogrammetry, Remote Sensing and Spatial Information Sciences, IV-2/W1, 241-248.

Edelsbrunner, H, Harer, J, 2010. Computational Topology: An Introduction. American Mathematical Society. 
Ekholm, A., Fridqvist, S., 2000. A concept of space for building classification, product modelling, and design. Automation in construction, 9, 315-328.

Fuentes-Pacheco, J., Ruiz-Ascencio, J., Rendón-Mancha, J. M., 2012. Visual simultaneous localization and mapping: a survey. Artificial Intelligence Review, 43, 55-81.

Groves, P. D., 2013. Principles of GNSS, inertial, and multisensor integrated navigation systems. 2nd edn, Artech House.

Huisman, O., de By, R. A., 2009. Principles of GIS. ITC Enschede.

Isikdag, U., Zlatanova, S., Underwood, J., 2013. A BIM-Oriented Model for supporting indoor navigation requirements. Computers, Environment and Urban Systems, $41,112-123$.

Khoshelham, K., Díaz-Vilariño, L., 2014. 3D modelling of interior spaces: Learning the language of indoor architecture. International Archives of the Photogrammetry, Remote Sensing and Spatial Information Sciences - ISPRS Archives, 40, 321-326.

Kosinov, S., Caelli, T., 2002. Inexact Multisubgraph Matching using Graph Eigenspace and Clustering Models. T. Caelli, A. Amin, R. P. W. Duin, D. de Ridder, M. Kamel (eds), Structural, Syntactic, and Statistical Pattern Recognition, Springer Berlin Heidelberg, Berlin, Heidelberg, 133-142.

Lee, J., Li, K., Zlatanova, S., Kolbe, T. H., Nagel, C., Becker, T., 2016. IndoorGML. http://docs.opengeospatial.org/is/14-005r4/14-005r4.html.

Leibniz, G. W., Clarke, S., Ariew, R., 2000. Leibniz and Clarke: Correspondence.

Lemmens, M. J. P. M., 2013. Indoor Positioning. GIM International, 5.

Luxburg, U. Von, 2007. A Tutorial on Spectral Clustering A Tutorial on Spectral Clustering. Statistics and Computing, 17, 395-416.

Marel, H. Van Der, 2016. Reference Systems for Surveying and Mapping, Lecture Notes. Delft University of Technology.

Mautz, R., 2012. Indoor Positioning Technologies. ETH Zurich, Department of Civil, Environmental an Geomatic Engineering.

Munch, E., 2017. A User's Guide to Topological Data Analysis. Journal of Learning Analytics, 4, 47-61.

Nourian, P., 2016. Configraphics: Graph theoretical methods for design and analysis of spatial configurations. Delft University of Technology, Delft.

Nourian, P., 2018. On Topology and Topological Data Models in Geometric Modeling of Space, Lecture Notes.

Nourian, P., Rezvani, S., Sariyildiz, I. S., Van Der Hoeven, F. D., 2016. Spectral modelling for spatial network analysis. Proceedings of the Symposium on Simulation for Architecture and Urban Design (simAUD 2016).
Pedregosa, F., Varoquaux, G., Gramfort, A., Michel, V., Thirion, B., Grisel, O., Blondel, M., Prettenhofer, P., Weiss, R., Dubourg, V., Vanderplas, J., Passos, A., Cournapeau, D., Brucher, M., Perrot, M., Duchesnay, E., 2011. Scikit-learn: Machine Learning in Python. Journal of Machine Learning Research, 12, 2825-2830.

Riisgaard, S., Blas, M. R., 2003. SLAM for Dummies. A Tutorial Approach to Simultaneous Localization and Mapping, 22.

Rowland, $\quad$ T., $2019 . \quad$ Manifold. http://mathworld.wolfram.com/Manifold.html.

Sithole, George, Zlatanova, Sisi, 2016. Position, Location, Place and Area: an Indoor Perspective. ISPRS Annals of Photogrammetry, Remote Sensing and Spatial Information Sciences, III-4, 89-96.

Spielman, D. A., 2007. Spectral Graph Theory and its Applications. 48th Annual IEEE Symposium on Foundations of Computer Science (FOCS'07), 29-38.

Stover, C., Weisstein, E. W., 2019. Euclidean Space. http://mathworld.wolfram.com/EuclideanSpace.html.

Weisstein, E. W., 2019a. Dual Graph. http://mathworld.wolfram.com/DualGraph.html.

Weisstein, E. W., 2019b. Metric Space. http://mathworld.wolfram.com/MetricSpace.html.

Weisstein, E. W., 2019c. Topology. http://mathworld.wolfram.com/Topology.html.

Wolfram-Alpha, 2019.

https://www.wolframalpha.com/input/?i=space.

Space.

Worboys, M. F., Duckham, M., 2004. GIS: A Computing Perspective. CRC press.

Xiao, J., Zhou, Z., Yi, Y., Ni, L. M., 2016. A Survey on Wireless Indoor Localization from the Device Perspective. ACM Computing Surveys, 49, 1-31.

Yassin, A, Nasser, Y, Awad, M, Al-Dubai, A, Liu, R, Yuen, C, Raulefs, R, 2016. Recent Advances in Indoor Localization: A Survey on Theoretical Approaches and Applications. IEEE Communications Surveys Tutorials, PP, 1.

Zafari, F., Gkelias, A., Leung, K., 2017. A Survey of Indoor Localization Systems and Technologies. 1-30.

Zlatanova, S., Liu, L., Sithole, G., Zhao, J., Mortari, F., 2014. Space subdivision for indoor applications. GISt Report No. 66, Delft University of Technology, OTB Research Institute for the Built Environment.

Zlatanova, S., Sithole, G., Nakagawa, M., Zhu, Q., 2013. Problems in indoor mapping and modelling. International Archives of the Photogrammetry, Remote Sensing and Spatial Information Sciences - ISPRS Archives, 40, 63-68. 